\title{
High Levels of IL-8 and MCP-1 in Cerebrospinal Fluid of COVID-19 Patients with Cerebrovascular Disease
}

\author{
Sebastián Guartazaca-Guerrero ${ }^{1,2 \dagger}$, Jahir Rodríguez-Morales ${ }^{1,2 \dagger}$, Salma A. Rizo-Téllez ${ }^{3,4}$, \\ Helena Solleiro-Villavicencio ${ }^{5}$, Aldo F. Hernández-Valencia ${ }^{2}$, José Damián Carrillo-Ruiz ${ }^{2,6}$, \\ Galileo Escobedo ${ }^{3 *}$ and Lucía A. Méndez-García ${ }^{3 *}$ \\ ${ }^{1}$ Especialidad en Neurocirugía, Facultad de Medicina, Universidad Nacional Autónoma de México, Coyoacán, Mexico \\ City 04510, ${ }^{2}$ Neurology and Neurosurgery Unit, General Hospital of Mexico "Dr. Eduardo Liceaga", Mexico City 06720, \\ ${ }^{3}$ Laboratory of Immunometabolism, Research Division, General Hospital of Mexico "Dr. Eduardo Liceaga", Mexico City \\ 06720, ${ }^{4}$ PECEM, Facultad de Medicina, Universidad Nacional Autónoma de México, Coyoacán, Mexico City 04510, \\ ${ }^{5}$ Posgrado en Ciencias Genómicas, Universidad Autónoma de la Ciudad de México, Plantel Del Valle, Benito Juárez, \\ Mexico City 03100, ${ }^{6}$ Facultad de Ciencias de la Salud, Universidad Anáhuac, Campus Norte, Huixquilucan,
} Estado de Mexico 52786, Mexico

The coronavirus family has tropism for the Central Nervous System (CNS), however, there is no solid evidence demonstrating that the neurological effects of COVID-19 result from direct viral infection or systemic inflammation. The goals of this study were to examine the cytokine profile and the presence of SARS-CoV-2 messenger ribonucleic acid (mRNA) in cerebrospinal fluids (CSF) from two patients with cerebrovascular disease and COVID-19. Although the SARS-CoV-2 mRNA was not detected in CSF of both patients, we found abnormally high levels of numerous proinflammatory cytokines and chemokines, especially IL-8 and MCP-1. Since these chemokines mediate activation and recruitment of neutrophils, monocytes, and macrophages, it is feasible that cerebrovascular disease related-neuroinflammation found in both patients results from an exacerbated inflammatory response instead of SARS-CoV-2 direct invasion to CNS. These results suggest that neuroinflammation plays a key role in cerebrovascular disease and COVID-19.

Key words: SARS-CoV-2, COVID-19, Cerebrospinal fluid, Cerebrovascular disease, IL-8, MCP-1

\section{INTRODUCTION}

The coronavirus disease 2019 (COVID-19) is a respiratory tract infection caused by the severe acute respiratory syndrome coronavirus 2 (SARS-CoV-2). Besides the lungs, COVID-19 also affects other organs such as kidneys, liver, heart, and brain [1]. Neurologi-

Submitted April 21,2021, Revised May 24, 2021,

Accepted June 8,2021

* To whom correspondence should be addressed.

Galileo Escobedo, TEL: 55-2789-2000, FAX: 55-5623-2669

e-mail:gescobedog@msn.com

Lucía A. Méndez-García, TEL: 55-2789-2000, FAX: 55-5623-2669

e-mail: angelica.mendez.86@hotmail.com

"These authors equally contributed to this work. cal alterations associated with SARS-CoV-2 infection include anosmia, headache, loss of consciousness, ischemic stroke, meningoencephalitis, and microhemorrhage [2]. The coronavirus family has tropism for the Central Nervous System (CNS) [3]. However, there is not yet solid evidence demonstrating that the neurological effects of COVID-19 result from direct viral infection or neuroinflammation associated with systemic inflammation and cytokine storm. Herein, we examine the cytokine profile of cerebrospinal fluids (CSF) from two patients with cerebrovascular disease concurrent with COVID-19, admitted to the Neurosurgery Department of the General Hospital of Mexico. We found high levels of numerous proinflammatory cytokines, especially interleukin (IL-) 8 and monocyte chemoattractant protein-1 (MCP-1). Although the presence of SARS-CoV-2 was confirmed in the airway epithelium by
Copyright $\odot$ Experimental Neurobiology 2021 www.enjournal.org
This is an Open Access article distributed under the terms of the Creative Commons Attribution Non-Commercial License (http://creativecommons.org/licenses/by-nc/4.0) which permits unrestricted non-commercial use, distribution, and reproduction in any medium, provided the original work is properly cited. 
real time-polymerase chain reaction (RT-PCR), we did not detect expression of viral particles in CSF of both patients.

\section{CASES REPORT}

\section{Case 1}

A 54-year old female, with no metabolic disorders or previous surgeries reported, and medical history of chronic obstructive pulmonary disease of 12 years of evolution, treated with nebulized salbutamol as needed. The relatives reported she had mild to moderated headache the last four days, balance impairment, and generalized weakness, although the presence of neurological symptoms associated to COVID-19 such as anosmia and dys- geusia were denied. She was admitted to emergency room with sudden loss of consciousness with the following vital signs: BP (110/80 mmHg), HR (80 bpm), BF (18 bpm), body temperature $\left(36.1^{\circ} \mathrm{C}\right.$ ), and a Glasgow Coma Scale (GCS) of 6 points (eye opening: 2 points, verbal response: 2 points, and motor response: 2 points) so we started airway management. The cranial computed tomography (CT) scan revealed cerebrovascular disease (CVD) characterized by acute intra-axial hemorrhage in the left cerebellar hemisphere associated with peripheral edema, which obliterated cortical subarachnoid spaces and cisternae of the posterior fossa and bulges the tentorium (Fig. 1A); hemorrhage extended into the fourth ventricle, which were displaced and compressed to the contralateral direction (Fig. 1A). Likewise, the hemorrhage extended
A

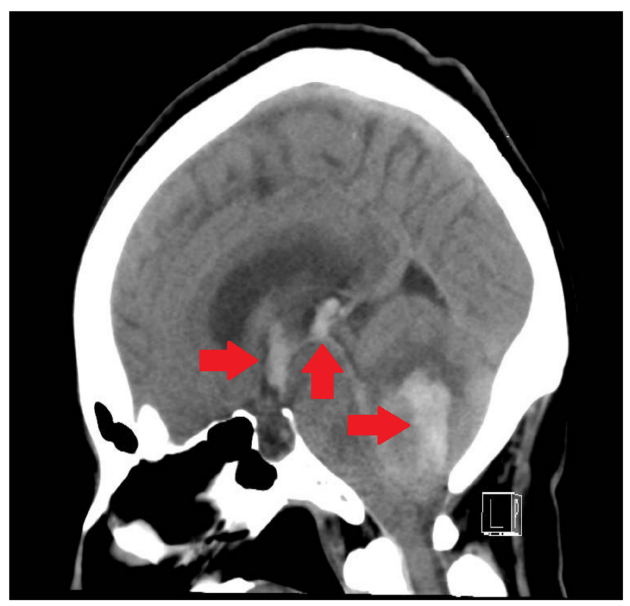

C

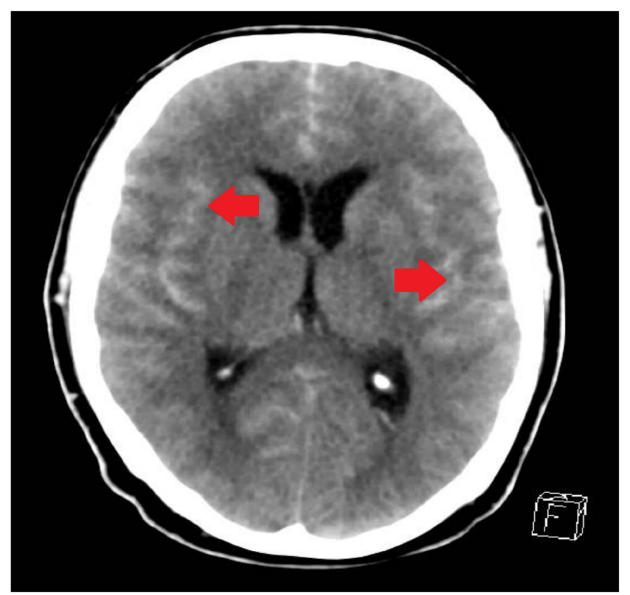

B

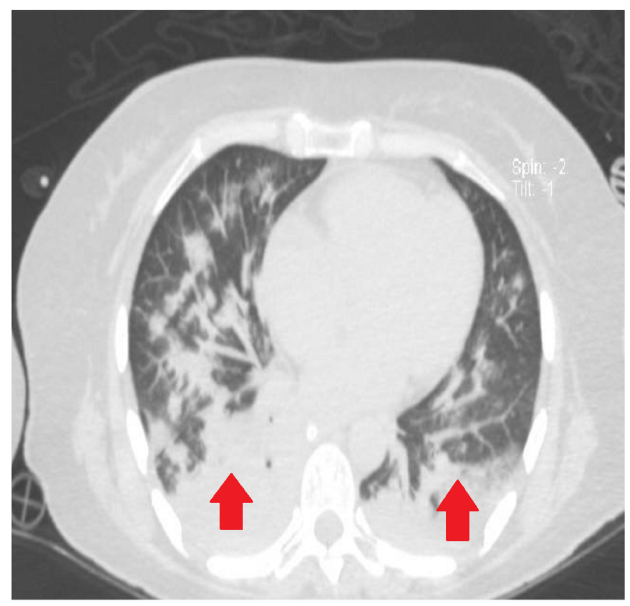

D

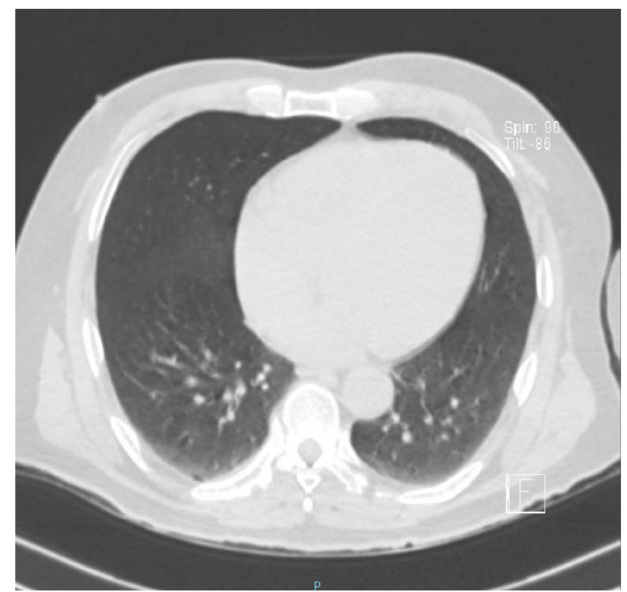

Fig. 1. Cranial and chest CT of patients with cerebrovascular disease and COVID-19. (A) cranial CT in sagittal reconstruction scan showing acute hemorrhage in the left cerebellar hemisphere with fourth ventricle compromise and basal cisternae obliteration, diffuse cerebral edema, obliterated subarachnoid space in posterior fose, and intraventricular hemorrhage. (B) Chest CT scan in Axial lung window showing several nodules with well-defined and irregular borders. (C) Cranial CT scan, at hospital admission, showing diffuse subarachnoid hemorrhage, spreading through both lateral sulcus predominantly to right side, and the perimesencephalic cisterns; incipient level in hematic range is also observed in the left ventricular inferior horn. (D) Chest CT scan in axial lung window showing homogenous lung parenchyma. Red arrows show sites of hemorrhage (A, C) and pulmonary infiltration (B). 
to the choroid plexuses of the supratentorial ventricles and led to obstructive hydrocephalus. The cortical subarachnoid spaces and supratentorial basal cisterns were obliterated (Fig. 1A). Measurement of laboratory parameters showed high leucocyte and neutrophil counts, whereas lymphocyte and monocyte counts decreased. She also presented high serum levels of glucose, total cholesterol, indirect bilirubin, gamma glutamyl transferase (GGT), creatinine kinase myocardial band (CK-MB), fibrinogen, D-dimer, and procalcitonin. There was no concluding evidence of COVID-19 in chest CT scan (CO-RADS III) (Fig. 1B), and then the presence of SARS-CoV-2 mRNA was confirmed by RT-PCR in nasopharyngeal swabs. Urgent right-sided precoronal ventriculostomy was performed. The patient presented clinical findings concurring with brain death immediately after postoperative period, and she died 24 hours later because of cardiopulmonary arrest.

\section{Case 2}

A 45-year-old male, with no metabolic disorders or previous surgeries, and medical history of ankylosing spondylitis diagnosed 23 years ago, under treatment with acetaminophen, diclofenac, and methylprednisolone. After reporting an undefined seizure accompanied by psychomotor agitation and disorientation one hour after awakening, he was admitted to the emergency room with vital signs at admission: BP (130/90 mmHg), HR (90 bpm), BF (20 bpm), and body temperature $\left(36.5^{\circ} \mathrm{C}\right.$ ) and GCS of 14 points (eye opening: 4 points, verbal response: 4 points, and motor response: 6 points). The relatives reported that the patient did not present any neurological symptom associated with COVID-19 such as anosmia or dysgeusia. The cranial computed tomography (CT) scan revealed CVD characterized by diffuse subarachnoid hemorrhage (Fisher IV), with WFNS I and HUNT \& HESS I (Fig. 1C). The cerebral parenchyma was heterogeneous due to the presence of hyperdense areas in the hematic range, the subarachnoid space, and the grooves of the parietal lobes that extended into the predominantly right Silvio fissures. The CT scan also showed increased density of interhemispheric fissure and round morphology image. The ventricular system was predominant at the temporal level, wherein incipient periventricular hypodensity and density in the hematic range on the left side were found. In digital cerebral angiography, a saccular aneurysm in the right A1 segment was evidenced. Twelve hours later, he presented sudden neurological impairment, thus we decided to perform a second cranial CT scan in which we found left middle cerebral artery stroke with areas of hemorrhage of acute-subacute stage at the left temporoparietal level and the body of the ipsilateral caudate nucleus. Hematic density inside the left lateral ventricle, at the level of the occipital horn was observed. Laboratory parameters revealed leukocytosis, lymphopenia, accompanied by high levels of fibrinogen and Ddimer concurring with SARS-CoV-2 infection (Table 1). Chest CT scan showed no typical anomalies (CO-RADS I) (Fig. 1D); however, RT-PCR in nasopharyngeal swabs confirmed the presence of SARS-CoV-2 mRNA. After two weeks of having received conservative treatment, the patient exhibited a negative RT-PCR test result for SARS-CoV-2. Then, left-sided pterional craniotomy

Table 1. Biochemical and immunological parameters of the patients

\begin{tabular}{|c|c|c|c|}
\hline Parameters & Case 1 & Case 2 & $\begin{array}{c}\text { Reference } \\
\text { range in CSF }\end{array}$ \\
\hline Glucose (mg/dl) & 161.00 & 122.00 & 74-106 \\
\hline Total cholesterol (mg/dl) & 236 & 175 & $<200$ \\
\hline Triglycerides (mg/dl) & 96 & 157 & $<150$ \\
\hline Indirect bilirubin (mg/dl) & 0.42 & 0.36 & $<0.2$ \\
\hline GGT (IU/L) & 50.00 & 57.00 & $\mathrm{M}<55 \mathrm{~W}<38$ \\
\hline CK-MB (IU/L) & 58.00 & 9.00 & $<24$ \\
\hline Fibrinogen (mg/dl) & 383.00 & 562.00 & $200-400$ \\
\hline Dimer-D $(\mathrm{Ug} / \mathrm{L})^{*}$ & 14,760 & 4,304 & $0-550$ \\
\hline Procalcitonin (ng/ml) & 0.06 & 0.06 & $<0.01$ \\
\hline Leucocytes $(\times 10 \mathrm{e} 3 / \mathrm{ul})$ & 12.10 & 15.70 & $4.5-10$ \\
\hline Neutrophils $(\times 10 \mathrm{e} 3 / \mathrm{ul})$ & 11.30 & 14.20 & $3-7$ \\
\hline Lymphocytes $(\times 10 \mathrm{e} 3 / \mathrm{ul})$ & 0.70 & 0.9 & $1-3$ \\
\hline Monocytes $(\times 10 \mathrm{e} 3 / \mathrm{ul})$ & 0.100 & 0.500 & $0.3-0.8$ \\
\hline $\mathrm{IL}-1 \beta(\mathrm{pg} / \mathrm{ml})$ & 18.95 & 10.00 & $<4$ \\
\hline IL-10 (pg/ml) & 32.38 & 9.79 & $2.82-5.30$ \\
\hline IL-13 (pg/ml) & 9.76 & 6.11 & Undetectable \\
\hline IL-6 (pg/ml) & 202.88 & 24.48 & 1.11 \\
\hline IL-12(pg/ml) & 322.99 & 15.87 & 3.58 \\
\hline RANTES (pg/ml) & 261.50 & 45.15 & $5.43-226.8$ \\
\hline Eotaxin $(\mathrm{pg} / \mathrm{ml})$ & 75.47 & 55.06 & 0.849 \\
\hline IL-17 (pg/ml) & 23.29 & 5.06 & $1-6$ \\
\hline MIP-1a (pg/ml) & 317.46 & 25.00 & $10.62 \pm 6.85$ \\
\hline GMC-SF (pg/ml) & 6.84 & 5.66 & Undetectable \\
\hline MIP- $1 \beta(\mathrm{pg} / \mathrm{ml})$ & 58.06 & 10.0 & $4.69 / 10.3$ \\
\hline MCP-1 $(\mathrm{pg} / \mathrm{ml})^{\star}$ & 15,000 & 337.51 & 160 \\
\hline IL-15 (pg/ml) & 296.44 & 45.00 & 3.51 \\
\hline IFN- $\gamma(\mathrm{pg} / \mathrm{ml})$ & 11.90 & 10.0 & $<0.3$ \\
\hline IFN- $\alpha(\mathrm{pg} / \mathrm{ml})$ & $2,129.62$ & 95.29 & 2.14 \\
\hline IL-1RA (pg/ml) & 382.11 & 16.71 & 25.00 \\
\hline TNF- $\alpha(\mathrm{pg} / \mathrm{ml})$ & 16.00 & 12.00 & $<4 / 1.51-2.11$ \\
\hline IL-2 $(\mathrm{pg} / \mathrm{ml})^{\star}$ & 4.51 & 3.00 & $70.62 \pm 1.1$ \\
\hline IL-7 (pg/ml) & 89.26 & 63.40 & 1.35 \\
\hline IP-10 (pg/ml) & 468.07 & 51.97 & $2.46-4.07$ \\
\hline IL-2R (pg/ml) & 56.68 & 7.11 & $28.2 \pm 3.2$ \\
\hline $\mathrm{MIG}(\mathrm{pg} / \mathrm{ml})$ & 20.46 & 9.99 & 6 \\
\hline IL-4 (pg/ml) & 31.19 & 3.87 & Undetectable \\
\hline IL-8 $(\mathrm{pg} / \mathrm{ml})^{\star}$ & $62,680.36$ & 377.67 & $67.5-96.4$ \\
\hline
\end{tabular}

${ }^{*}$ The most apparent differences between cases and reference range are shown.

GGT, gamma-glutamyl transpeptidase; CK-MB, creatine kinase MB; IL, interleukine; MIP 1 $\alpha / \beta$, Macrophage Inflammatory Protein 1-Alpha/Beta; GMC-SF, Granulocyte Macrophage Colony-Stimulating Factor; MCP-1, monocyte chemoattractant protein-1; IFN- $\gamma / \alpha$, interferon gamma/alpha; TNF-a, tumor necrosis factor-alpha; IP-10, interferon gamma-induced protein 10; MIG, monokine induced by interferon-gamma. 
and aneurysm clipping surgery was performed. He was hospital discharged due to improvement two weeks after surgery, with normal vital signs as follows: $\mathrm{BP}$ (120/70 mmHg), HR (70 bpm), BF (18 bpm), and body temperature $\left(36.5^{\circ} \mathrm{C}\right)$.

Both patients were admitted to the emergency department because of neurological symptoms rather than respiratory complications associated with SARS-CoV-2 infection. The relatives of the patients provided written informed consent, previously approved by the Institutional Ethical committee of the General Hospital of Mexico, which guaranteed that the study was conducted in rigorous adherence to the principles described in the 1964 Declaration of Helsinki and its posterior amendment in 2013. Cranial and chest CT scans were obtained from the Carestream Viu Motion software v.12.1.5.7 from the digital electronic file of the General Hospital of Mexico.

The CSF sample was obtained during surgery by ventricular shunt for case 1, whereas the CSF sample was obtained by lumbar puncture at admission for case 2. Viral particles of SARS-CoV-2 including spike $S 1$ protein $(S)$, nucleocapsid (N), envelope (E), and RNA-dependent RNA polymerase (RdRp) were analyzed in CSF by RT-PCR using the next primer sequences: $\mathrm{S}$ (forward: GCTGGTGCTGCAGCTTATTA; reverse: AGGGTCAAGTGCACAGTCTA ), N (forward: CAATGCTGCAATCGTGCTAC; reverse: GTTGCGACTACGTGATGAGG ), E (forward: TTCGGAAGAGACAGGTACGTTA; reverse: AGCAGTACGCACACAATCG ), RdRp (forward: AGAATAGAGCTCGCACCGTA; reverse: CTCCTCTAGTGGCGGCTATT), $18 \mathrm{~S}$ (forward: CGGCTACCACATCCAAGGAA; reverse: GCTGGAATTACCGCGGCT). All tests were performed following the next thermocycling steps: $95^{\circ} \mathrm{C}$ for 10 minutes, $95^{\circ} \mathrm{C}$ for 15 seconds, $60 \sim 63^{\circ} \mathrm{C}$ for 30 seconds (depending on the primer sequence), $72{ }^{\circ} \mathrm{C}$ for 45 seconds, and a final extension step of $72^{\circ} \mathrm{C}$ for 7 minutes, after reaching 50 cycles.

Although RT-PCR test in nasopharyngeal swabs confirmed the SARS-CoV-2 infection in Case $1(C T=35.09)$ and Case 2 $(\mathrm{CT}=35.17)$, the expression of $\mathrm{S}, \mathrm{N}, \mathrm{E}$, and RdRp RNA was not detected in CSF from both patients.

The cytokine analysis was performed using the Human Cytokine Magnetic 25-Plex Panel (Life technologies, Frederick, MD, USA) following the manufacturer's instructions. Results were analyzed in the MILLIPLEX ${ }^{\mathrm{TM}}$ Analyst 5.1 Flex software. CSF samples from both patients revealed that levels of IL-6, IL-12, eotaxin, IL-17, MIP-1a, GMC-S, MIP-1b, MCP-1, IL-15, IFN- $\gamma$, IFN- $\alpha$, TNF- $\alpha$, IL-7, MIG, and IL-8 were dramatically higher than those found in reference values. However, IL-8 and MCP-1 showed the most impressive elevations in Case 1 as compared to Case 2. In fact, the Case 1 exhibited 166- and 44-fold increases in IL-8 and MCP-1, respectively, with respect to Case 2. IL-2 was the only cytokine below reference values (Table 1).

\section{DISCUSSION}

Cases with SARS-CoV-2 infection-related neurological complications are increasing; thus a thorough evaluation of the CNS should be considered in patients with COVID-19. Therefore, patients should provide CSF biopsy, which is a minimally invasive form to obtain a CNS tissue sample. The presence of SARSCoV-2 in CSF has been documented only in a few COVID-19 patients with neurological symptoms [4]. In this sense, Meinhardt and coworkers proposed that SARS-CoV-2 may enter the CNS via the neural-mucosal interface in olfactory mucosa in the nose, allowing its detection in CSF by means of RT-PCR [5]. In parallel, another study in mice reported that spike S1 protein can cross the blood brain barrier, reaching the CSF of the CNS [6]. However, in this study we were not able to detect the SARS-CoV-2 in CSF by RT-PCR, even though the same molecular test in nasopharyngeal swabs confirmed the presence of SARS-CoV-2 in the airway epithelium of both patients. Nevertheless, the fact that we did not detect SARS-CoV-2 mRNA in CSF samples does not confirm the absence of the virus in the CNS and further studies are needed. On the contrary, the cytokine storm was clearly evidenced in CSF, which presumably associates with the neurological symptoms accompanying COVID-19 [7].

Recently, Mousa-Ibrahim and cols [8] reported intracranial hemorrhage as well as thrombosis within six COVID-19 patients. Moreover, in an exceptional review study, Pramitasuri and cols. show that ischemic stroke is more frequent than intracerebral hemorrhage in SARS-CoV-2 patients. However, ischemic stroke in the context of SARS-CoV-2 infection more frequently evolved to hemorrhage than conventional ischemic stroke, which associates with increased mortality and worst outcome [9].

The relationship between SARS-CoV-2 infection and CVD lies in the ability of the virus to lead to hypercoagulable states that in turn increases fibrin deposition and D-dimer elevation [10]. Upon binding to the angiotensin-converting-enzyme (ACE2) receptor in brain capillary endothelial cells, SARS-CoV-2 causes cerebral endothelial damage that in turn leads to the development of intracranial hemorrhage, release of subintimal collagen, and platelet aggregation associated with clot production [11]. In the present study, we did not evaluate directly the brain capillary endothelium; however, we found no evidence of SARS-CoV-2 mRNA in CSF from both patients by RT-PCR. Therefore, it is feasible that cerebrovascular events observed in these patients were caused by an exacerbated proinflammatory response in the CNS. In this sense, 
our data show that both patients had intracranial hemorrhages presumably related to COVID-19, characterized by increased CSF levels of cytokines associated with CNS inflammation such as IL$1 \beta$, IL- 6 , TNF- $\alpha$ and IFN- $\gamma$, and extremely high concentrations of IL- 8 and MCP- 1 .

IL-8 also referred to as CXCL8, is an important chemoattractant of neutrophils with the ability to eliminate cells infected with virus or bacteria, which in turn leads to collateral tissue injury. In a normal physiological state, IL-8 is scantly expressed in the CNS; however, IL-8 increases in several neurological pathologies such as ischemic brain injury, stroke, and traumatic brain injury. After ischemic brain damage, IL-8 increases first in CSF, inducing elevation of this cytokine in plasma, which results in systemic inflammation and persistent damage to CNS [12]. Likewise, MCP1 also referred to as CCL2, is an important chemokine with the ability to recruit a wide variety of leukocytes such as macrophages and monocytes toward damaged tissues [13]. Similarly to IL-8, increased MCP-1 levels have been involved in neurological pathologies such as multiple sclerosis (MS), cerebral stroke, and traumatic brain injury [12].

Previous studies in Alzheimer Disease (AD) show that brain microvessels can release high levels of thrombin and IL-8, promoting the formation of neutrophil extracellular traps (NETs). In cerebral capillaries of AD patients, thrombin induces the expression of IL-8, thus contributing to the intravascular NETosis. Likewise, the MCP-1/CCR2 axis promotes migration of monocytes to the vascular endothelium of the CNS with the aim of clearing amyloid peptides in AD patients [14]. Despite the function of IL-8 and MCP-1 in the COVID-19-related cytokine storm has been widely studied, the role of these cytokines in the neurological dysfunction associated with COVID-19 is not fully elucidated.

Neutrophilia is a recurrent hallmark of acute infection in COVID-19 patients that leads to the formation of NETs, which are composed by networks of DNA, histones, and proteolytic enzymes that normally function in response to pathogens. Histones of NETs activate the coagulative kallikrein-kinin system and platelets, which present the High Mobility Group protein B1 (HMGB1) to neutrophils, stimulating NET formation. Once NETs bind to the endothelium, they compromise its integrity by the recruitment of more neutrophils, producing endothelial damage $[15,16]$.

Interestingly, in patients with ischemic strokes due to COVID-19 the role of NETs has a pivotal importance. Additionally, it is believed that neuroinflammation observed in stroke-related COVID-19 associates with the cytokine storm, wherein IL- $1 \beta$ appears to play a pivotal role. IL- $1 \beta$ is able to induce the expression of IL-17 and IL-6, which in turn can interact with astrocytes, microglia, and neurons, intensifying the cytokine storm and favoring the recruit- ment of neutrophils and macrophages to cerebral parenchyma via HMGB1 protein [17]. In this scenario, IL-8 and MCP-1 might also contribute to neuroinflammation by recruiting neutrophils and macrophages to the vascular endothelium of the brain, favoring endothelial damage, NET formation, and the hypercoagulable state.

Despite we cannot precise whether high levels of IL-8 and MCP1 are a cause or consequence of CVD in COVID-19 patients, this information supports the role of these cytokines in aggravating the severity of the intracranial hemorrhages in patients with SARSCoV-2 infection.

Identification of the cytokine profile in CSF of patients with CVD concurrent with COVID-19 may allow starting a timely anti-inflammatory pharmacological approach. The use of drugs that inhibit the IL- 8 and MCP-1-dependent signaling pathways may help reduce exacerbation of the inflammatory response in patients with COVID-19, thus decreasing the magnitude of the neurological damage. For this reason, we propose to conduct clinical trials aimed to test the efficacy of Tofacinitib and Reparaxyn in hyper-inflamed COVID-19 patients. Tofacinitib is an inhibitor of the JAK-STAT signaling pathway that indirectly obstructs the action of proinflammatory cytokines and chemokines such as IL6, IL-8, MCP-1, MMP3, and MMP2/9 [18]. In parallel, Reparaxyn is an inhibitor of CXC chemokines receptor types 1 (CXCR1) and 2 (CXCR2), which becomes more relevant in patients with COVID-19 that show marked neutrophilia.

In conclusion, we outlined the cytokine profile in CSF of patients with CVD concurrent with COVID-19. Our findings suggest that CNS damage may result from the excessive inflammatory response mediated by IL- 8 and MCP-1, instead of being caused by direct infection of SARS-CoV-2 to the CNS.

\section{ACKNOWLEDGEMENTS}

SGG and JRM are medical residents of the Programa de Residencias Médicas en la Especialidad de Neurocirugía de la Facultad de Medicina de la Universidad Nacional Autónoma de México (UNAM). SRT is a doctoral student from the Plan de Estudios Combinados en Medicina, Licenciatura y Doctorado (PECEM) of the Universidad Nacional Autónoma de México (UNAM) and has received CONACYT fellowship 762603. This project was partially financed by the Research Division, General Hospital of Mexico "Dr. Eduardo Liceaga" with the registration number DI/20/501/03/8.

\section{REFERENCES}

1. Gavriatopoulou M, Korompoki E, Fotiou D, Ntanasis-Statho- 
poulos I, Psaltopoulou T, Kastritis E, Terpos E, Dimopoulos MA (2020) Organ-specific manifestations of COVID-19 infection. Clin Exp Med 20:493-506.

2. Deigendesch N, Sironi L, Kutza M, Wischnewski S, Fuchs V, Hench J, Frank A, Nienhold R, Mertz KD, Cathomas G, Matter MS, Siegemund M, Tolnay M, Schirmer L, Pröbstel AK, Tzankov A, Frank S (2020) Correlates of critical illness-related encephalopathy predominate postmortem COVID-19 neuropathology. Acta Neuropathol 140:583-586.

3. Matías-Guiu J, Gomez-Pinedo U, Montero-Escribano P, Gomez-Iglesias P, Porta-Etessam J, Matias-Guiu JA (2020) Should we expect neurological symptoms in the SARSCoV-2 epidemic? Neurologia (Engl Ed) 35:170-175.

4. Domingues RB, Mendes-Correa MC, de Moura Leite FBV, Sabino EC, Salarini DZ, Claro I, Santos DW, de Jesus JG, Ferreira NE, Romano CM, Soares CAS (2020) First case of SARS-COV-2 sequencing in cerebrospinal fluid of a patient with suspected demyelinating disease. J Neurol 267:31543156.

5. Meinhardt J, Radke J, Dittmayer C, Franz J, Thomas C, Mothes R, Laue M, Schneider J, Brünink S, Greuel S, Lehmann M, Hassan O, Aschman T, Schumann E, Chua RL, Conrad C, Eils R, Stenzel W, Windgassen M, Rößler L, Goebel HH, Gelderblom HR, Martin H, Nitsche A, Schulz-Schaeffer WJ, Hakroush S, Winkler MS, Tampe B, Scheibe F, Körtvélyessy P, Reinhold D, Siegmund B, Kühl AA, Elezkurtaj S, Horst D, Oesterhelweg L, Tsokos M, Ingold-Heppner B, Stadelmann C, Drosten C, Corman VM, Radbruch H, Heppner FL (2021) Olfactory transmucosal SARS-CoV-2 invasion as a port of central nervous system entry in individuals with COVID-19. Nat Neurosci 24:168-175.

6. Rhea EM, Logsdon AF, Hansen KM, Williams LM, Reed MJ, Baumann KK, Holden SJ, Raber J, Banks WA, Erickson MA (2021) The S1 protein of SARS-CoV-2 crosses the bloodbrain barrier in mice. Nat Neurosci 24:368-378.

7. Farhadian S, Glick LR, Vogels CBF, Thomas J, Chiarella J, Casanovas-Massana A, Zhou J, Odio C, Vijayakumar P, Geng B, Fournier J, Bermejo S, Fauver JR, Alpert T, Wyllie AL, Turcotte C, Steinle M, Paczkowski P, Dela Cruz C, Wilen C, Ko AI, MacKay S, Grubaugh ND, Spudich S, Barakat LA (2020) Acute encephalopathy with elevated CSF inflammatory markers as the initial presentation of COVID-19. BMC Neu$\operatorname{rol} 20: 248$

8. Mousa-Ibrahim F, Berg S, Od TPDetola O, Teitcher M, Ruland S (2021) Intracranial hemorrhage in hospitalized SARS-CoV-2 patients: a case series. J Stroke Cerebrovasc Dis 30:105428.

9. Pramitasuri TI, Laksmidewi AAAP, Putra IBK, Dalimartha FA (2021) Neutrophil extracellular traps in coronavirus disease-19-associated ischemic stroke: a novel avenue in neuroscience. Exp Neurobiol 30:1-12.

10. Kihira S, Schefflein J, Pawha P, Rigney B, Delman BN, Xuan D, Fifi J, Doshi AH, Belani P (2021) Neurovascular complications that can be seen in COVID-19 patients. Clin Imaging 69:280-284.

11. Baig AM, Khaleeq A, Ali U, Syeda H (2020) Evidence of the COVID-19 virus targeting the CNS: tissue distribution, hostvirus interaction, and proposed neurotropic mechanisms. ACS Chem Neurosci 11:995-998.

12. Semple BD, Kossmann T, Morganti-Kossmann MC (2010) Role of chemokines in CNS health and pathology: a focus on the CCL2/CCR2 and CXCL8/CXCR2 networks. J Cereb Blood Flow Metab 30:459-473.

13. David BA, Kubes P (2019) Exploring the complex role of chemokines and chemoattractants in vivo on leukocyte dynamics. Immunol Rev 289:9-30.

14. Pietronigro EC, Della Bianca V, Zenaro E, Constantin G (2017) NETosis in Alzheimer's disease. Front Immunol 8:211.

15. Al-Anazi KA, Al-Anazi WK, Al-Jasser AM (2020) Neutrophils, NETs, NETosis and their paradoxical roles in $\mathrm{CO}$ VID-19. J Stem Cell Ther Transplant 4:003-010.

16. Bonaventura A, Vecchié A, Abbate A, Montecucco F (2020) Neutrophil extracellular traps and cardiovascular diseases: an update. Cells 9:231.

17. Manda-Handzlik A, Demkow U (2019) The brain entangled: the contribution of neutrophil extracellular traps to the diseases of the central nervous system. Cells 8:1477.

18. Gao W, McGarry T, Orr C, McCormick J, Veale DJ, Fearon U (2016) Tofacitinib regulates synovial inflammation in psoriatic arthritis, inhibiting STAT activation and induction of negative feedback inhibitors. Ann Rheum Dis 75:311-315. 